\title{
An In-Depth Review of Niraparib in Ovarian Cancer: Mechanism of Action, Clinical Efficacy and Future Directions
}

\author{
Melek Akay · Ionut-Gabriel Funingana · Grisma Patel • \\ Rami Mustapha $\cdot$ Ernese Gjafa • Tony Ng $\cdot$ Kenrick Ng (D) · \\ Michael J. Flynn
}

Received: June 21, 2021 / Accepted: July 22, 2021 / Published online: August 7, 2021

(C) The Author(s) 2021

\section{ABSTRACT}

Niraparib is an oral, potent, highly selective poly-ADP ribose polymerase 1 (PARP1) and PARP2 inhibitor. In most developed countries, it is approved as a maintenance treatment for epithelial ovarian, fallopian tube, or primary peritoneal cancer in patients with complete or partial response to platinum-based therapy.

\section{Akay}

Department of Medical Oncology, St George's

University Hospitals NHS Foundation Trust,

London, UK

I.-G. Funingana

Department of Medical Oncology, University of

Cambridge, Cambridge, UK

G. Patel $\cdot$ K. Ng $\cdot$ M. J. Flynn

Department of Medical Oncology, University

College London Hospitals, London, UK

R. Mustapha · T. Ng

School of Cancer and Pharmaceutical Sciences,

King's College London, London, UK

R. Mustapha · T. Ng

Cancer Research UK King's Health Partners Centre, London, UK

E. Gjafa

Department of Medical Oncology, Barts Health NHS

Trust, London, UK

\section{T. $\mathrm{Ng} \cdot \mathrm{K} . \mathrm{Ng}(\square)$}

Cancer Institute, University College London, 72

Huntley Street, London, UK

e-mail: K.ng@ucl.ac.uk
These approvals are based on results of randomised, double-blind, placebo-controlled trials, particularly the NOVA trial and more recently the PRIMA trial. In this comprehensive review, we delve into the scientific basis of PARP inhibition, discussing both preclinical and clinical data which have led to the current approval status of niraparib. We also discuss ongoing trials and biological rationale of combination treatments involving niraparib, with particular focus on antiangiogenic drugs, immune checkpoint inhibitors and cyclic GMPAMP synthase stimulator of interferon genes (cGAS/STING) pathway. In addition, we reflect on potential strategies and challenges of utilising current biomarkers for treatment selection of patients to ensure maximal benefit.

Keywords: Combined therapy; Niraparib; Ovarian Cancer; PARP inhibitors; Pharmacokinetics 


\section{Key Summary Points}

Niraparib is a potent and highly selective poly-ADP ribose polymerase (PARP) inhibitor.

It is approved as a maintenance treatment for epithelial ovarian cancer which has demonstrated response to platinum chemotherapy.

We review the scientific basis of PARP inhibition, chronologically detailing preclinical and clinical data which have led to the approvals to date.

We also discuss ongoing trials and the biological rationale for combination treatments, with a focus on the cGAS/ stimulator of interferon genes (STING) pathway and immune checkpoint inhibitors.

The review also highlights practical challenges faced by clinicians, such as limitations of current strategies in defining biomarkers, particularly with regard to homologous recombination deficiency status.

\section{INTRODUCTION}

DNA damage can occur in a range of different ways through a variety of endogenous and exogenous stressors. Because of the diversity of lesions, a number of complex, sophisticated repair pathways have evolved which work synergistically to repair DNA damage. These include base excision repair (BER), nucleotide excision repair and mismatch repair for singlestrand breaks (SSB), and homologous recombination (HR) or non-homologous end-joining (NHEJ) for repair of double-strand breaks (DSBs) [1].

The family of poly-ADP ribose polymerases (PARPs) are enzymes which mediate repair of DNA SSBs primarily through BER. PARP1, the founding and most abundant family member, senses SSBs and binds to DNA at the site of damage. Upon binding, it catalyses the transfer of ADP-ribose units to many proteins, including PARP1 itself, to form long poly-ADP ribose (PAR) chains, a process known as poly-ADP-ribosylation (PARylation). The auto-PARylation of PARP1 triggers its release from the site of damage, enabling DNA repair factors, which have been recruited by the PAR chains, to localise to the lesion [2]. The majority of PARPdependent repair activity is through PARP1, with PARP2 contributing only approximately $10 \%$ [3].

\section{PROMOTING SYNTHETIC LETHALITY THROUGH PARP INHIBITION}

Simultaneous inhibition of PARP1 and PARP2 results in persistence of SSBs. There is evidence to suggest that this is due not only to inhibition of the catalytic repair of SSB induced by PARP1, but also to PARP1 trapping [4]. PARP inhibitors (PARPi) block the synthesis of PAR chains, thereby preventing autoPARylation of PARP1 and its release from the site of DNA damage. The trapped PARP1 prevents repair of SSBs, allowing progression to replication fork collapse and formation of DNA DSBs. A schematic of the mechanism of action of PARPi is shown in Fig. 1.

This results in a heavy reliance on DSB repair pathways such as HR. PARP inhibition in patients with a defective HR pathway can therefore result in gross genomic instability and ultimately cell death by apoptosis. BRCA 1 and BRCA2 are key proteins involved in HR; therefore, in theory, PARP inhibition should be selectively potent against cells which have biallelic BRCA1 or BRCA2 deficiency. Thus, knockout of either BRCA or PARP alone is associated with cell viability, while simultaneous knockout of both genes results in cell death, a concept known as 'synthetic lethality' [5]. There are many other genes that are also crucial for HR and therefore have a synthetic lethal interaction with PARP, including FANC, ATM, CHEK2, MRE11A and RAD51 [6]. Many sporadic 

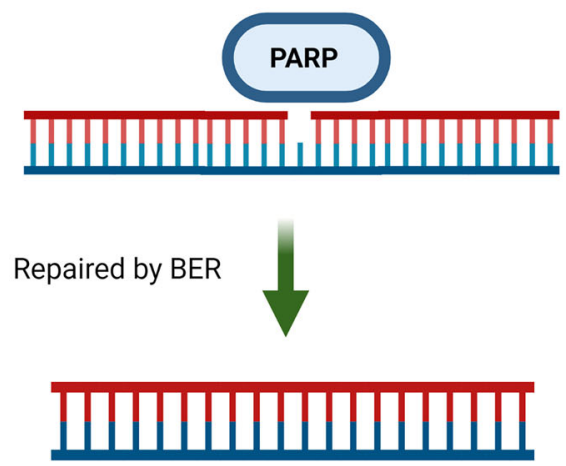

\section{Survival}
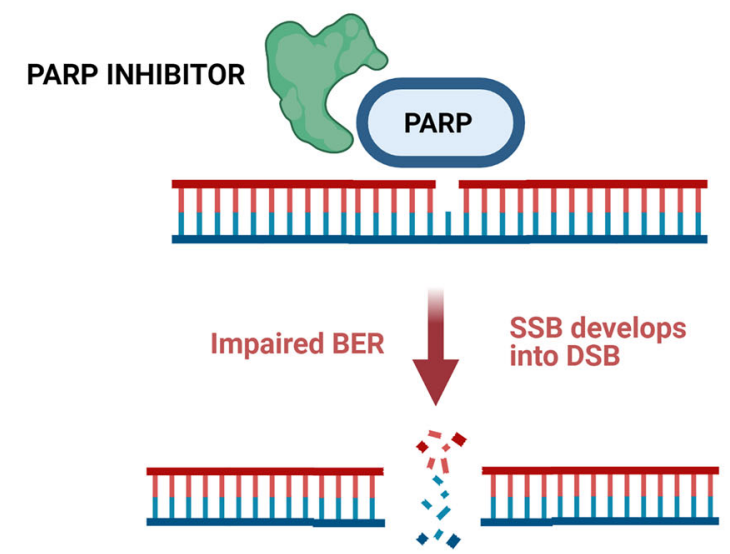

HR proficient
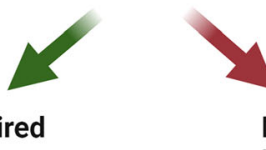

HR deficient

DSB repaired

DSB accumulation and replication fork collapse

Cell Death

Fig. 1 Mechanism of action of PARP inhibitors. In the presence of base excision repair and homologous recombination deficiency, double-strand break accumulation

cancers harbour disruption of these genes, and the resulting homologous recombination deficiency (HRD) can therefore serve as a predictive biomarker for sensitivity to PARP inhibition.

This article aims to provide a comprehensive review of niraparib, covering the scientific basis of PARP inhibition, discussing both preclinical and clinical data which have led to the current approval status of the drug. The review is based on previously conducted studies and does not contain any studies with human participants or animals performed by any of the authors.

\section{DEVELOPMENT OF NIRAPARIB}

Niraparib is an oral, potent, highly selective PARP1 and PARP2 inhibitor. Its discovery as the compound 2- ${ }^{7}-2 \mathrm{H}$-indazole-7-carboxamide 56
Table 1 Inhibitory activity of niraparib on individual PARP isoforms [7]

\begin{tabular}{ll}
\hline & IC $_{\mathbf{5 0}}(\mathbf{n M})$ \\
\hline PARP1 & 3.8 \\
PARP2 & 2.1 \\
PARP3 & 1300 \\
V-PARP & 330 \\
\hline
\end{tabular}

IC50 half-maximal inhibitory concentration

(or MK-4827) was first reported in 2009 [7]. Jones et al. assessed the activity of niraparib in vitro using an assay that measures the amount of PAR chains formed in cells as a result of DNA damage induced by hydrogen peroxide. Niraparib was demonstrated to have excellent 
potency against PARP1 and PARP2, with halfmaximal inhibitory concentrations $\left(\mathrm{IC}_{50}\right)$ of 3.8 and $2.1 \mathrm{nM}$, respectively, and at least 100 -fold selectivity over other members of the PARP family (Table 1 ).

In vitro inhibition of proliferation of BRCA mutated cancer cell lines was demonstrated, with $50 \%$ cytotoxic concentration $\left(\mathrm{CC}_{50}\right)$ of $18 \mathrm{nM}$ in BRCA1-deficient cells and $\mathrm{CC}_{50}$ of $90 \mathrm{nM}$ in BRCA2-deficient cells. Normal human prostate and mammary epithelial cells were resistant, with anti-proliferative effects seen at micromolar concentrations, indicating selective cytotoxicity for BRCA mutant cancer cells compared to surrounding tissue [7]. In vivo activity of niraparib on BRCA1 mutant cells was assessed using mice models with implanted human mammary adenocarcinoma cells that had been silenced for BRCA1 [8]. At a dose of $80 \mathrm{mg} / \mathrm{kg}$ niraparib, significant inhibition of tumour growth was observed after only 1-2 weeks of treatment, and continuous daily dosing for $>4$ weeks resulted in complete and sustained tumour regression. Activity against BRCA2-deficient cells was assessed using a pancreatic cancer cell xenograft model. At the same dose $(80 \mathrm{mg} / \mathrm{kg}$ niraparib), approximately $60 \%$ inhibition of tumour growth was observed after 2 weeks. In both cases, it was considered well tolerated, with $<10 \%$ reduction in body weight.

Penetration of the blood-brain barrier has also been assessed in the preclinical setting [9]. Pharmacokinetic studies were conducted in germline BRCA mutant intracranial xenograft models, and concentration-time profiles of niraparib in the brain were similar to those in plasma, with a mean brain-to-plasma

Table 2 Tumour growth inhibition of niraparib in xenograft models

\begin{tabular}{lll}
\hline $\begin{array}{l}\text { Dose of } \\
\text { niraparib }\end{array}$ & \multicolumn{2}{l}{ Tumour growth inhibition (\%) } \\
\cline { 2 - 3 } & $\begin{array}{l}\text { Subcutaneous } \\
\text { tumours }\end{array}$ & $\begin{array}{l}\text { Intracranial } \\
\text { tumours }\end{array}$ \\
\hline $15 \mathrm{mg} / \mathrm{kg}$ & 13 & 55 \\
$30 \mathrm{mg} / \mathrm{kg}$ & 49 & 56 \\
$45 \mathrm{mg} / \mathrm{kg}$ & 54 & 83 \\
\hline
\end{tabular}

concentration ratio of $0.85-0.99$ of the brain $T_{\max }$. Clinical activity in the brain was also assessed using a xenograft model with BRCA mutant pancreatic tumour cells implanted either subcutaneously or intracranially. Tumour growth inhibition values at different doses of niraparib are shown in Table 2 . The data support an intracranial anti-tumour effect and suggest that niraparib can effectively penetrate the central nervous system to exert a therapeutic benefit.

\section{PHASE 1 TRIAL}

In 2013, the results were published for a first-inhuman study of niraparib in patients with solid organ tumours across three sites, one in the UK and two in the USA [6]. One hundred patients in total were enrolled, 49 of whom had ovarian or primary peritoneal cancer, and 20 of whom had germline BRCA mutations and were evaluable radiologically. The proportion of patients achieving a RECIST [Response Evaluation Criteria In Solid Tumors] partial response (PR) was highest in those with BRCA mutated platinumsensitive disease, as demonstrated in Table 1 . The data suggest that both BRCA deficiency and platinum sensitivity can serve as predictive biomarkers for response to niraparib. The antitumour activity seen amongst patients with BRCA wild-type (BRCAwt) disease was felt to potentially reflect HRD due to somatic silencing of key genes (Table 3).

In terms of safety, the study demonstrated good tolerance of niraparib up to the recommended phase 2 dose of $300 \mathrm{mg} /$ day. Nonhaematological toxicities such as nausea and

Table 3 Objective response rate in patients with ovarian and primary peritoneal cancer to niraparib treatment by BRCA mutation and platinum status

\begin{tabular}{lll}
\hline & \multicolumn{2}{l}{ Objective response rate $(\boldsymbol{n}$ /total, \%) } \\
\cline { 2 - 3 } & BRCA mutated & BRCA wild-type \\
\hline Platinum-sensitive & $5 / 10(50 \%)$ & $1 / 3(33 \%)$ \\
Platinum-resistant & $3 / 9(33 \%)$ & $1 / 19(5 \%)$ \\
\hline
\end{tabular}


fatigue were rated as mild to moderate and were felt to be manageable. Haematological toxicity seemed to be dose-dependent, with anaemia being most common and reaching grade 3 or worse in $10 \%$ of patients. Thrombocytopenia was less common, but grade 4 thrombocytopenia was dose-limiting at $400 \mathrm{mg} /$ day. Neutropenia was the least common, affecting $24 \%$ of patients and reaching grade 3 in $4 \%$ of patients at doses of 300 or $400 \mathrm{mg}$. In general, myelosuppression was self-limiting and resolved with treatment breaks or dose reductions. The study concluded that overall, niraparib was well tolerated and demonstrated promising anti-tumour activity in both BRCA mutated and sporadic solid organ cancers.

\section{NOVA: THE FIRST THERAPEUTIC TRIAL FOR NIRAPARIB}

On the basis of these findings, a phase 3 randomised controlled trial designed to further evaluate efficacy of niraparib was undertaken. This was the pivotal phase 3 NOVA trial [10]. Eligibility criteria incorporated the findings of the phase 1 study, stipulating that patients must have completed their last dose of platinumbased therapy no more than 8 weeks prior to enrolment and must have demonstrated sensitivity to the penultimate platinum treatment regimen defined as a PR or CR for at least 6 months. BRCA mutation and HR status were not a feature of the inclusion criteria; however, patients were stratified according to the presence or absence of a germline BRCA mutation. Given there were some patients with sporadic ovarian cancer who also responded to niraparib in the phase 1 study, tumour samples from patients lacking a germline BRCA mutation were analysed for the presence of another mutational process causing HRD. The Myriad Genetics myChoice assay was used to detect these processes (discussed later). The study design is shown in Fig. 2.

The trial demonstrated a significant improvement in progression-free survival (PFS) in the niraparib group compared to placebo across all three populations, with HR of 0.27 (95\% CI 0.17-0.41) in the germline BRCA mutation (gBRCAmut) cohort, 0.45 (95\% CI 0.34-0.61) in the non-gBRCAmut cohort and 0.38 (95\% CI 0.24-0.59) in the HRD subgroup of the non-gBRCA cohort. Exploratory analyses performed on the HRD subgroup to assess whether somatic BRCA mutations were driving the observed benefit from niraparib showed a similar result in the somatic BRCAm group as the gBRCA cohort, HR 0.27 (95\% CI 0.08-0.9), while those with BRCAwt in the HRD subgroup benefitted less (HR 0.38; 95\% CI 0.23-0.63). The group which benefitted the least was the HRproficient (HRP) subgroup (HR 0.58, 95\% CI 0.36-0.92). However, even in this group, approximately $20 \%$ of the patients had a longterm benefit ( $>18$ months) from niraparib. On the basis of these findings, niraparib was granted global approval for the maintenance treatment of recurrent ovarian cancer regardless of HR or BRCA status, the first in its class to be licensed in this setting.

An updated analysis of the NOVA trial [11] was presented in the Society for Gynaecologic Oncology (SGO) Conference in 2021 and suggested an increase in median overall survival (OS) by 9.7 months in the BRCA-mutated population receiving niraparib, while no difference was seen in patients with non-germline-BRCA mutations. This needs to be interpreted in the context that OS was a secondary end point, and the study was not appropriately powered to demonstrate this.

Some of the most common adverse events of niraparib include anaemia, neutropenia, thrombocytopenia, nausea and fatigue. These have also been seen in other phase 3 trials of PARP inhibitors used in the maintenance setting, such as olaparib and rucaparib. Within the constraints of cross-trial comparisons, severe haematological toxicities are more common in niraparib (25\%) than in rucaparib and olaparib (19\% each) [12].

The updated analysis revealed that myelodysplastic syndrome or acute myeloid leukemia (MDS/AML) was reported in 3.5\% patients treated with niraparib, with slightly higher incidence in those with gBRCAm. It also showed that the most common adverse events (myelosuppression, nausea, fatigue, insomnia) seemed to occur mostly in the first few months, 


\section{NOVA: Study Design}

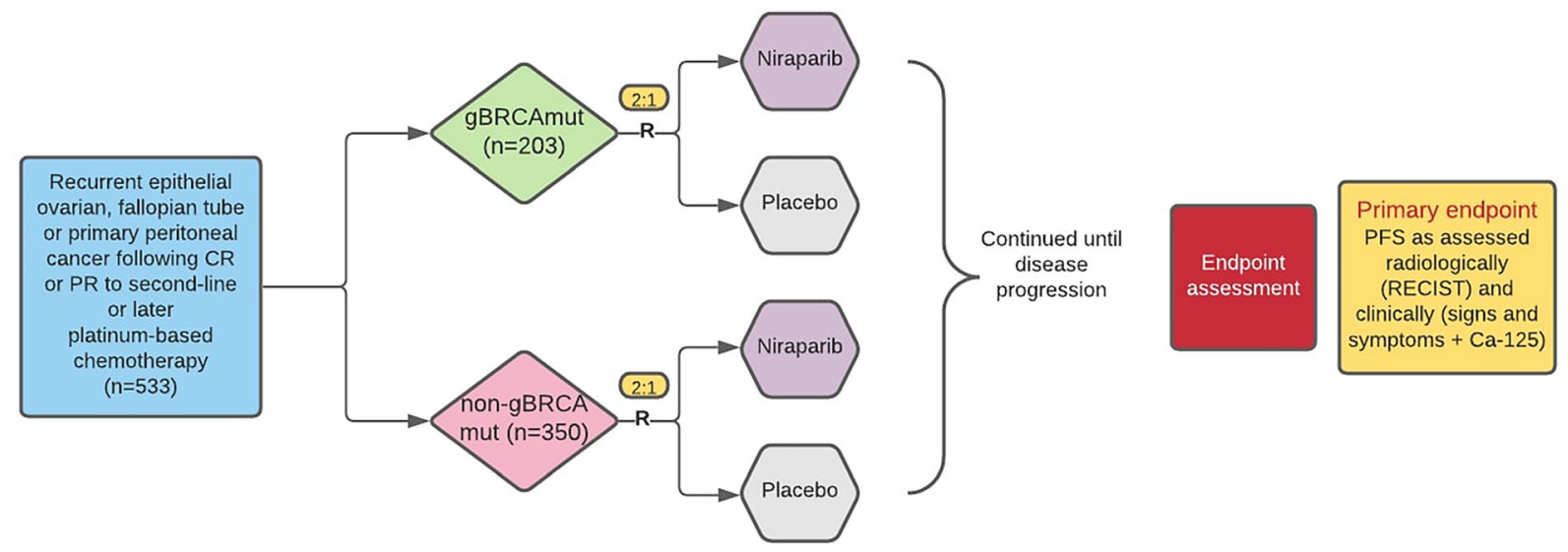

Fig. 2 NOVA study design. $C R$ complete response, $P R$ partial response, PFS progression-free survival, RECIST Response Evaluation Criteria in Solid Tumours,
gBRCAmut $=$ germline BRCA mutation, non-gBRCAmut $=$ No germline BRCA mutation with dose reductions being highest in month 1 and declining every month thereafter, highlighting the importance of appropriate dose reduction according to toxicity criteria.

In light of the toxicity profile, a retrospective analysis was carried out to identify parameters that could predict dose reductions. Patients with a body weight of $<77 \mathrm{~kg}$ and platelet count of $<150 \times 10^{9} / \mathrm{L}$ were found to have increased risk of treatment-emergent adverse events and haematological toxicity; therefore, a starting dose of $200 \mathrm{mg}$ has been recommended for patients with either of these parameters. Clinical efficacy did not appear to be affected by dose modifications in patients with dose reduction [13].

Importantly, quality-of-life measurements for patients receiving niraparib were similar to those for patients receiving placebo, which is particularly relevant in the maintenance setting [14].

\section{PRIMA TRIAL: MOVING NIRAPARIB TO THE FIRST-LINE MAINTENANCE SETTING}

While the NOVA study looked at patients with recurrent, heavily pretreated ovarian cancer, the PRIMA study was a phase 3 trial evaluating niraparib in the front-line setting in newly diagnosed ovarian cancer. [15] The study design is shown in Fig. 3.

The primary end point was median PFS and a significant improvement was demonstrated in both the overall population and the HRD subgroup (HR 0.62; 95\% CI 0.5-0.76 in overall population and 0.43 ; $95 \%$ CI $0.31-0.59$ in the HRD group).

Exploratory analyses performed on the HRD subgroup suggested that there was significant benefit in those with BRCA mutations (HR 0.40, 95\% CI 0.27-0.62) as well as in those without BRCA mutations (HR 0.50, 95\% CI 0.31-0.83). Overall, again, the smallest margin of benefit was observed in those with HRP disease (HR 


\section{PRIMA: Study Design}

Phase III trial of niraparib as front-line maintenance therapy following front-line platinum-based chemotherapy in patients with advanced ovarian cancer

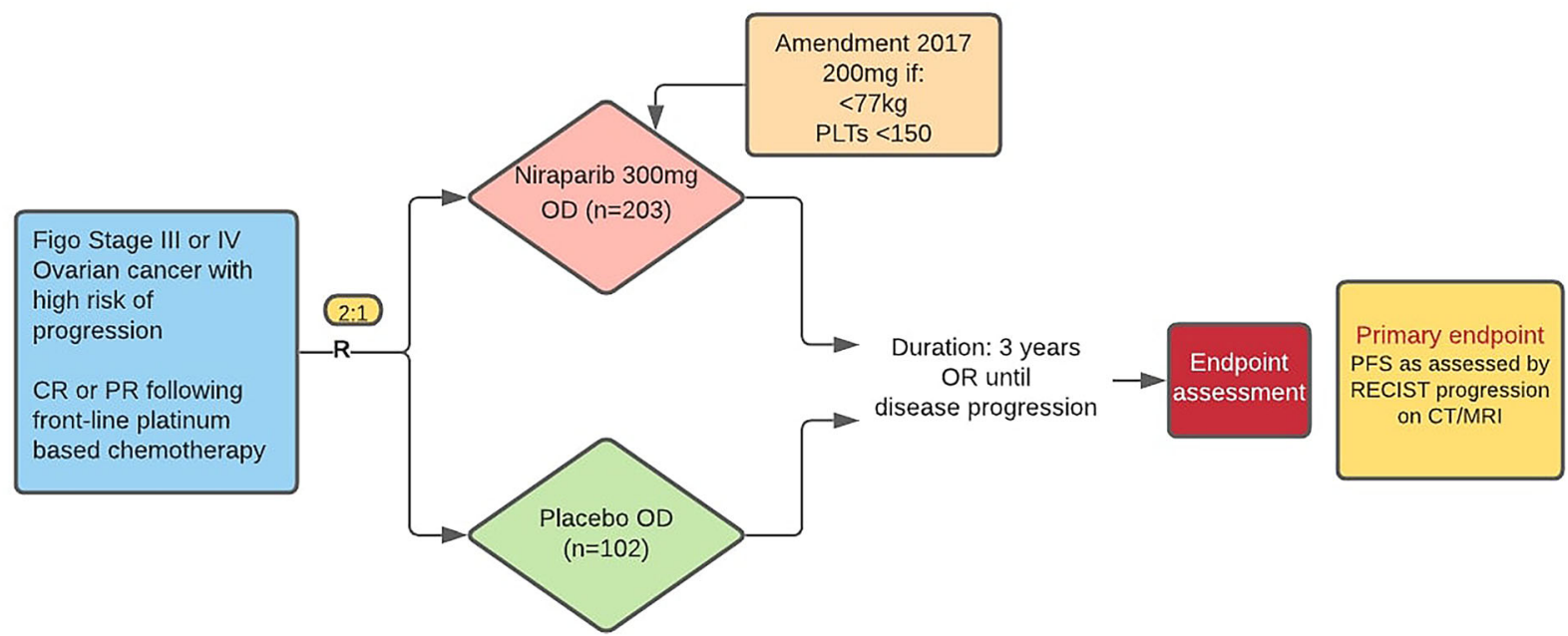

Fig. 3 PRIMA study design. $C R$ complete response, $P R$ partial response, $P F S$ progression-free survival, $R E C I S T$ Response Evaluation Criteria in Solid Tumours, $O D$ once daily, Plt platelets

Table 4 High-risk features for patients with newly diagnosed ovarian cancer

Inoperable FIGO stage III disease

Suboptimally debulked $(>1 \mathrm{~cm})$ stage III disease

Stage IV disease

Receipt of neoadjuvant chemotherapy

Partial response to first-line platinum-based

chemotherapy

FIGO International Federation of Gynecology and Obstetrics

0.68, 95\% CI 0.49-0.94). However, it is important to note that the study was powered only for the predefined primary analysis population, which was the overall study population and the HRD subgroup.

In comparison to other trials of up-front PARP inhibitor maintenance (for example the SOLO-1 trial of olaparib), the PRIMA study population included a high proportion of highrisk patients (Table 4), with $67 \%$ requiring neoadjuvant chemotherapy, 23\% with residual disease after primary de-bulking surgery and $31 \%$ with only a PR to platinum-based chemotherapy.

PRIMA concluded that niraparib maintenance after first-line platinum-based chemotherapy can extend benefit to all patients, with lower sensitivity amongst the HRP population. The results of the study formed the evidence basis for approval of niraparib as the only oral, once-daily PARPi as monotherapy maintenance treatment for women with firstline platinum-responsive advanced ovarian cancer, regardless of biomarker status, in both the USA and EU, and more recently the United Kingdom (Fig. 4).

\section{QUADRA TRIAL: INVESTIGATING THE ROLE OF PARPI AS MONOTHERAPY IN A HEAVILY PRETREATED SETTING}

While NOVA and PRIMA looked at niraparib as maintenance therapy, QUADRA was a 


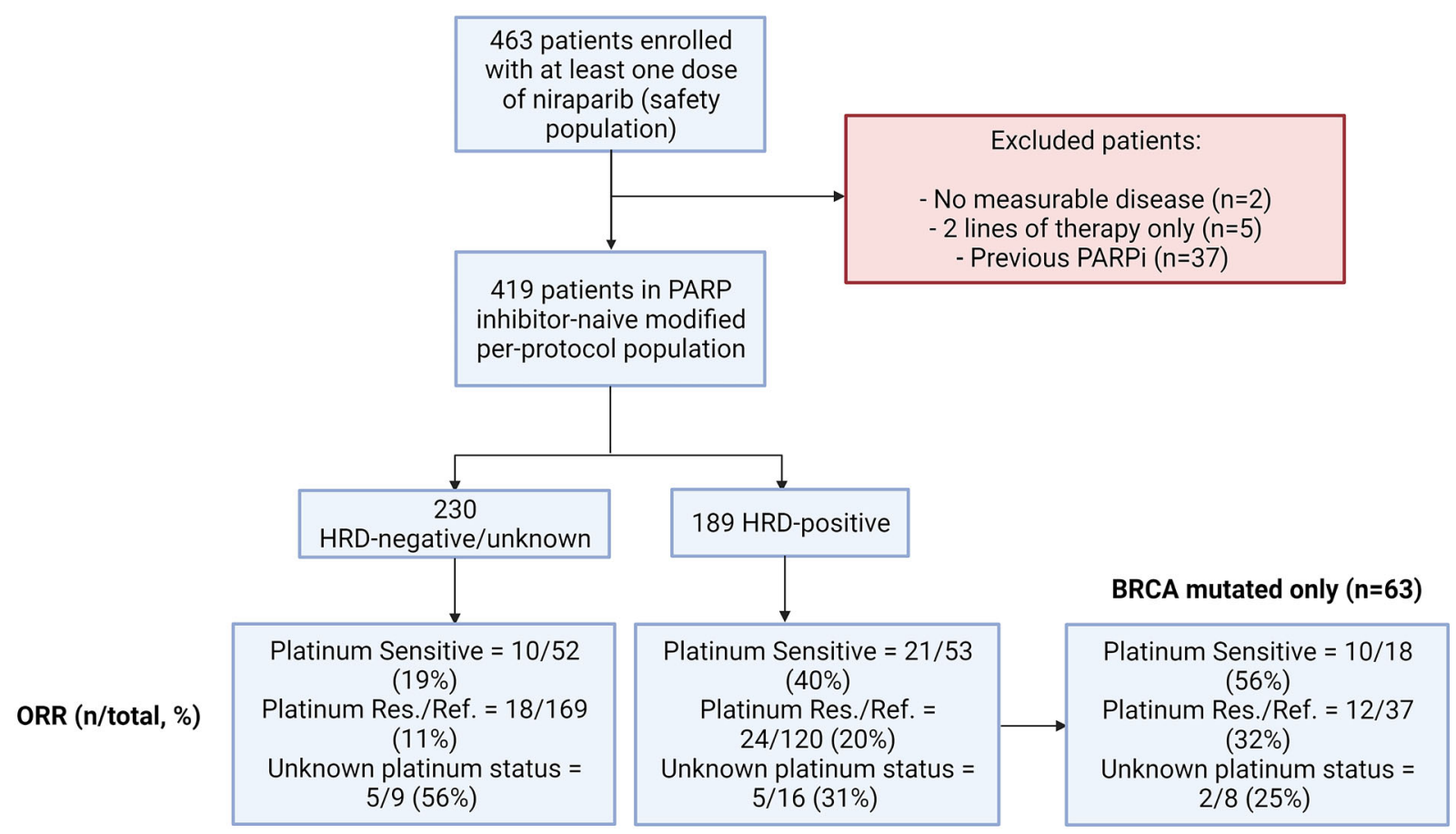

Fig. 4 QUADRA trial profile and overall response rates by HRD and platinum status. Platinum Res/Ref platinum chemotherapy-resistant or -refractory, PARPi PARP inhibitor

multicentre, single-arm phase 2 study which investigated niraparib as a treatment line in heavily pretreated patients with ovarian cancer ( $\geq 3$ lines of chemotherapy) [16]. Unlike NOVA or PRIMA, QUADRA initially did not exclude platinum-resistant or platinum-refractory disease; however, the eligibility criteria were subsequently amended to restrict enrolment to those with a response to first-line platinumbased therapy of $>6$ months. Thirty-three percent of the 463 patients enrolled were platinumresistant (platinum-free interval of 1-6 months after most recent platinum-based therapy) and $35 \%$ platinum-refractory (platinum-free interval $<28$ days). The study met its primary end point, with $28 \%$ of the primary efficacy population (47 patients with platinum-sensitive HRD tumours who had received 3-4 lines of therapy) achieving an overall response. Subgroup analyses (for which the study was not powered) demonstrated a hierarchical spectrum of benefit based on biomarker and platinum status, the results of which are shown in Fig. 4. The pattern demonstrated suggests that BRCA mutation is most predictive of a response to niraparib, even more so than platinum sensitivity. The data for median OS support this, with OS of 26 months in the BRCAm population, 19 months in the HRD population and 15.5 months in the HRP population.

On the basis of QUADRA, the Food and Drug Administration (FDA) approved niraparib as monotherapy for patients with advanced ovarian, fallopian tube or primary peritoneal cancer who have had $\geq 3$ previous lines of treatment and whose cancer is HRD-positive. Niraparib is not currently indicated for this setting in the UK or EU. A timeline of approvals for niraparib in the USA, UK and Europe is shown in Fig. 5.

\section{DEFINING 'HRD' STATUS: LIMITATIONS OF CURRENT STRATEGIES}

The results from the three monotherapy niraparib trials indicate a clear hierarchy of sensitivity, with the BRCA mutated cancers standing to benefit the most and the HRP cancers 


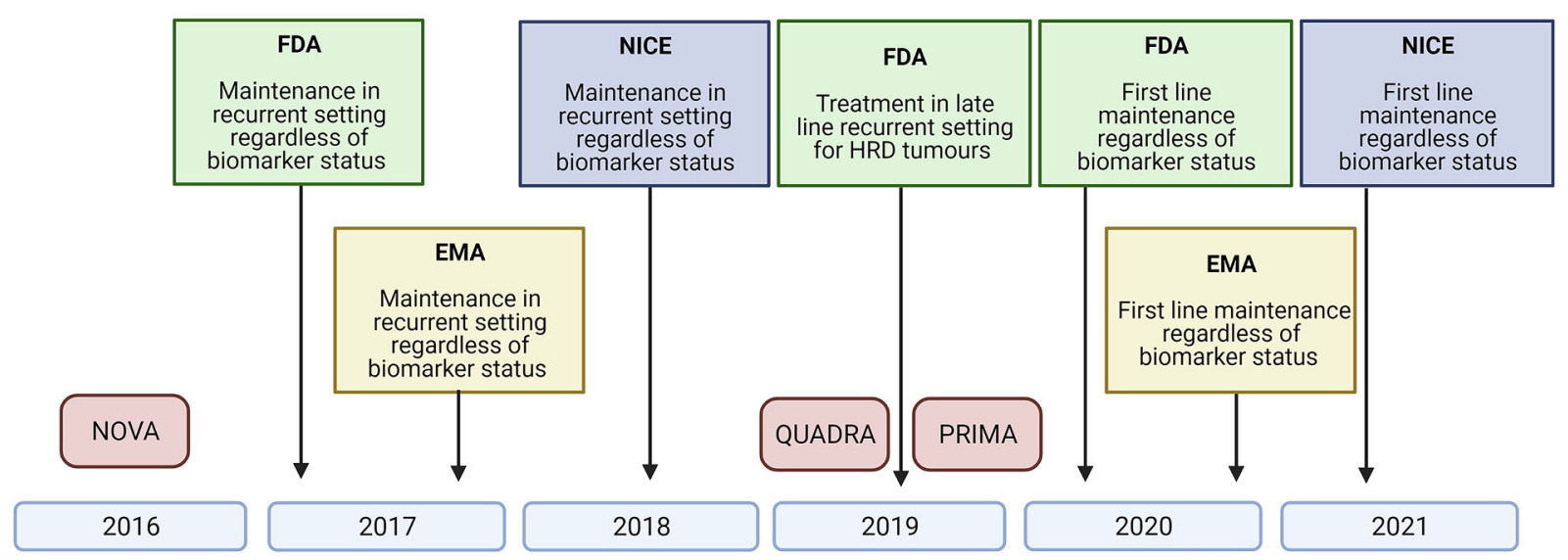

Fig. 5 Key dates for the FDA, EMA and NICE approvals for niraparib. FDA Food and Drug Administration, EMA European Medicines Agency, NICE National Institute for Health and Care Excellence

benefitting the least. The fact that all three trials demonstrated some degree of benefit for patients with HRP tumours may reflect a limitation of the HRD assay used in distinguishing genuine HR deficiency and proficiency. The assay used in all three trials was the Myriad Genetics myChoice test. This is a next-generation sequencing (NGS) test that combines identification of germline or somatic BRCA1 or BRCA2 variants with quantification of genomic instability of the tumour [15]. Rather than detecting inactivation of specific genes in the HR repair pathway, the idea is to quantify chromosomal abnormalities indicative of HRD irrespective of the specific cause, a so called 'genomic scar'. This genomic instability is measured on the basis of loss of heterozygosity (LOH), telomeric allelic imbalance (TAI) and large-scale state transitions (LSTs). LOH is a feature of many cancers, describing the second hit to a tumour suppressor gene that already has one defective allele. TAI and LSTs are both features of HRD cells with TAI resulting from attempts to repair DNA DSBs using the errorprone NHEJ pathway and LSTs describing large chromosomal breaks. These three biomarkers are then used to generate a genomic instability score (GIS) with HR deficiency being defined by a score of greater than or equal to 42 or presence of a BRCA mutation [17]. Another commercially available genomic scar test is the FoundationOne assay, which uses NGS to determine percentage of genomic LOH, with 'LOH-high' indicating HRD positivity.

According to European Society for Medical Oncology (ESMO) recommendations [18], HRD tests which incorporate scores of allelic imbalance (GIS or LOH) can identify a subgroup of BRCAwt, platinum-sensitive cancers that derive a greater magnitude of benefit from PARPi therapy in some settings. However, the clinical utility of these tests is limited by their inability to consistently identify a BRCAwt subgroup that derives no benefit from PARPi [18]. Thus, the positive results seen amongst the so-called HRP population in the niraparib monotherapy trials throws into question the efficacy of the HRD test and it is likely that there is some degree of error with a proportion of BRCAwt patients being incorrectly labelled as HRP (or HRD-negative). In addition, it is important to note that these commercial HRD tests have been developed and validated by trials which have largely specified platinum-sensitivity as an inclusion criterion. This will inevitably have led to a potential selection bias in the context of the treatment-naïve or platinum-refractory or platinum-resistant patient, creating an area of large unmet clinical need. Finally, the HRD assays currently available do not provide a current estimate of HRD status, being valid only for the time at which the sample was obtained [18]. Thus, further work is needed to optimise testing 
to provide an accurate and real-time estimate of HR status.

The distinction of HRD and HRP is becoming more crucial going forward going forward as the treatment pathways for these two subgroups of patients are likely to diverge. The challenge will be to develop ways of enhancing the innate sensitivity of those with HRD disease and to overcome the innate resistance of those with HRP disease. Combination therapy offers a means of achieving this and several combinations with different targeted therapies are under investigation.

\section{COMBINATION THERAPIES}

Ovarian cancer is one of the most genomically complex of all solid tumours. The heterogeneity of ovarian cancer means that it is unlikely to be cured with single-agent therapy. Combination therapy is more likely to yield greater activity with longer duration, as the disease is likely to recur with potentially new resistance mechanisms. The genomic complexity, alongside intra-tumoural heterogeneity, has led clinicians to explore different angles to develop the most effective combination treatments alongside PARPi.

\section{Bevacizumab}

The lymph-angiogenic tumour microenvironment is an important element that has been a target for modulation in ovarian cancer.

Bevacizumab, an anti-vascular epithelial growth factor monoclonal antibody, has been licensed as first-line therapy alongside chemotherapy for advanced ovarian cancer for some time. The evidence for its use came from two large phase 3 randomised trials: GOG 0218 [19] and ICON7 [20]. Both studies demonstrated a significant improvement in PFS with maintenance bevacizumab versus placebo after platinum-based chemotherapy, although this improvement did not extend to OS, with the exception of those with high-risk disease in the ICON7 trial.

Vascular epithelial growth factor (VEGF) has been demonstrated to promote HR by contributing to the expression and function of RAD51, an essential enzyme in the HR pathway [21]. Inhibition of VEGF therefore leads to defective HR and could theoretically sensitize tumours to PARP inhibition, providing a rationale for this combination.

The phase 3 PAOLA-1 study randomised patients with newly diagnosed platinum-sensitive ovarian cancer to maintenance olaparib plus bevacizumab versus placebo plus bevacizumab [22]. A clear benefit was seen in the olaparib arm overall (HR 0.59, 95\% CI $0.49-0.72$ ) and in the BRCAm subgroup (HR $0.31 ; 95 \%$ CI $0.2-0.47$ ). The benefit of maintenance olaparib plus bevacizumab was clearly seen in HRD-positive tumours including BRCA mutations (HR 0.33, 95\% CI 0.25-0.45) and also in those with HRD-positive tumours without BRCA mutations (HR 0.43, 95\% CI 0.28-0.66). Once again, those with HRP tumours or HR status unknown derived the smallest margin of benefit (HR of $0.92,95 \%$ CI 0.71-1.17).

The phase 2 AVANOVA trial [23] randomised patients with platinum-sensitive, heavily pretreated recurrent ovarian cancer to niraparib alone or niraparib plus bevacizumab as a treatment line. Results showed a median PFS of 11.9 months with the combination of niraparib/bevacizumab versus 5.5 months with single-agent niraparib (adjusted HR 0.35; 95\% CI 0.21-0.57). This improvement was seen regardless of HRD positivity (HR 0.38, 95\% CI $0.20-0.72$ ) or negativity (HR $0.40,95 \%$ CI $0.19-0.85)$. These results led investigators to further evaluate this combination and move it to the front-line setting with the OVARIO study [24].

OVARIO is a phase 2, single-arm study of maintenance niraparib plus bevacizumab in a similar population as PAOLA-1: patients with advanced ovarian cancer who have responded to first-line platinum chemotherapy with bevacizumab. Preliminary results were recently presented at the SGO conference 2021 and demonstrated 18 -month PFS rates of $62 \%$ in the overall population, $76 \%$ amongst the HRD patients and $47 \%$ amongst the HRP patients. No new safety signals were generated, and the researchers concluded that the combination is 
safe and continues to prolong PFS for a majority of patients.

\section{Immune Checkpoint Inhibitors}

Immune checkpoint inhibitors (ICIs) have revolutionised the treatment of multiple solid tumours. At the time of writing, ICIs have not achieved comparable success in epithelial ovarian cancer, particularly in the context of monotherapy [25]. Nevertheless, we postulate that the combination of PARP inhibitors and immune checkpoint inhibitors is a biologically sound and synergistic treatment strategy.

To explore this potential synergy in detail, we need to review the cGAS/STING pathway which underscores the immunomodulatory effects of PARP inhibitors [26]. The cGAS-cGAMP-STING pathway is activated when cytosolic DNA is sensed by cyclic GMP-AMP synthase (cGAS). The mechanisms by which this cytosolic DNA accumulates are not fully understood, but include chromosome instability, DNA damage and oncosuppressor gene mutation or deletion in tumour cells [27]. PARP1 trapping induced by PARPi has also been proposed to generate cytosolic dsDNA and thus contribute to activation of this innate immune signalling pathway [28].

Upon sensing cytosolic DNA, cGAS catalyses the formation of cyclic GMP, which in turn leads to activation of stimulator of interferon genes (STING). This results in recruitment of TANK-binding kinase 1 (TBK1), followed by phosphorylation and activation of IRF3, causing the activation of type I IFN transcription [29]. The upregulation of type 1 interferons and other pro-inflammatory cytokines leads to tumour suppressive effects. Figure 6 provides a schematic of the activation of the cGAS/STING pathway by PARP inhibition.

In addition, cancer cells can induce activation of cGAS-STING signalling in nearby dendritic cells. There are several main hypotheses [30] to explain this phenomenon:

1. Tumour-derived cGAMP is secreted into the extracellular space and imported into host immune cells through the folate transporter SLC19A1 and direct activation of STING.
2. Tumour-derived DNA is released and transported into dendritic cell cytosol through unestablished mechanisms, and sensed by c-GAS STING pathway (e.g., indirect exosome-mediated DNA transfer [31]).

3. cGAMP transfer through gap junctions between cancer and immune cells [32].

Either way, the resultant production of type 1 interferons in the tumour microenvironment promotes dendritic cell maturation and crosspresentation of tumour-associated antigens to CD8+ T cells, leading to anti-tumour responses [29].

The combination of PARPi with an immune checkpoint inhibitor is a potent strategy for several reasons. PARPi have been shown to enhance PD-L1 expression on tumour cells both in vitro and in vivo, an effect which has been observed with both BRCA-deficient and BRCAproficient cell lines [33]. In theory, this could lead to an upregulation of the inhibitory PD-1/ PD-L1 axis which would dampen cytotoxic T cell response. Combining PD-1/PD-L1 inhibition with PARP inhibition would mitigate this mechanism of resistance, ensuring that the downstream immunomodulatory effects of PARP inhibitors are preserved.

In recent years, many in vitro and in vivo models have supported the use of this combination. In the preclinical setting, Wang et al. combined niraparib with anti-PD-1/anti-PD-L1 therapy and demonstrated enhanced immune cell infiltration, interferon-stimulated gene expression and tumour responses, irrespective of BRCA mutation status [34].

In BRCA1-deficient ovarian cancer mice models, Ding et al. found that PARP inhibition led to an anti-tumour immune response dependent on STING pathway activation [35]. They demonstrated that olaparib increased the number of intra-tumoural effector $\mathrm{CD}^{+}$and $\mathrm{CD}^{+} \mathrm{T}$ cells, associated with increased recruitment of dendritic cells, and significantly reduced myeloid-derived suppressor cells in the tumour tissue, spleen and blood. This response occurred via activation of the STING pathway in dendritic cells, mediated by sensing of tumourderived DNA and/or cGAMP [35]. They also discovered increased expression of PD-L1 on 


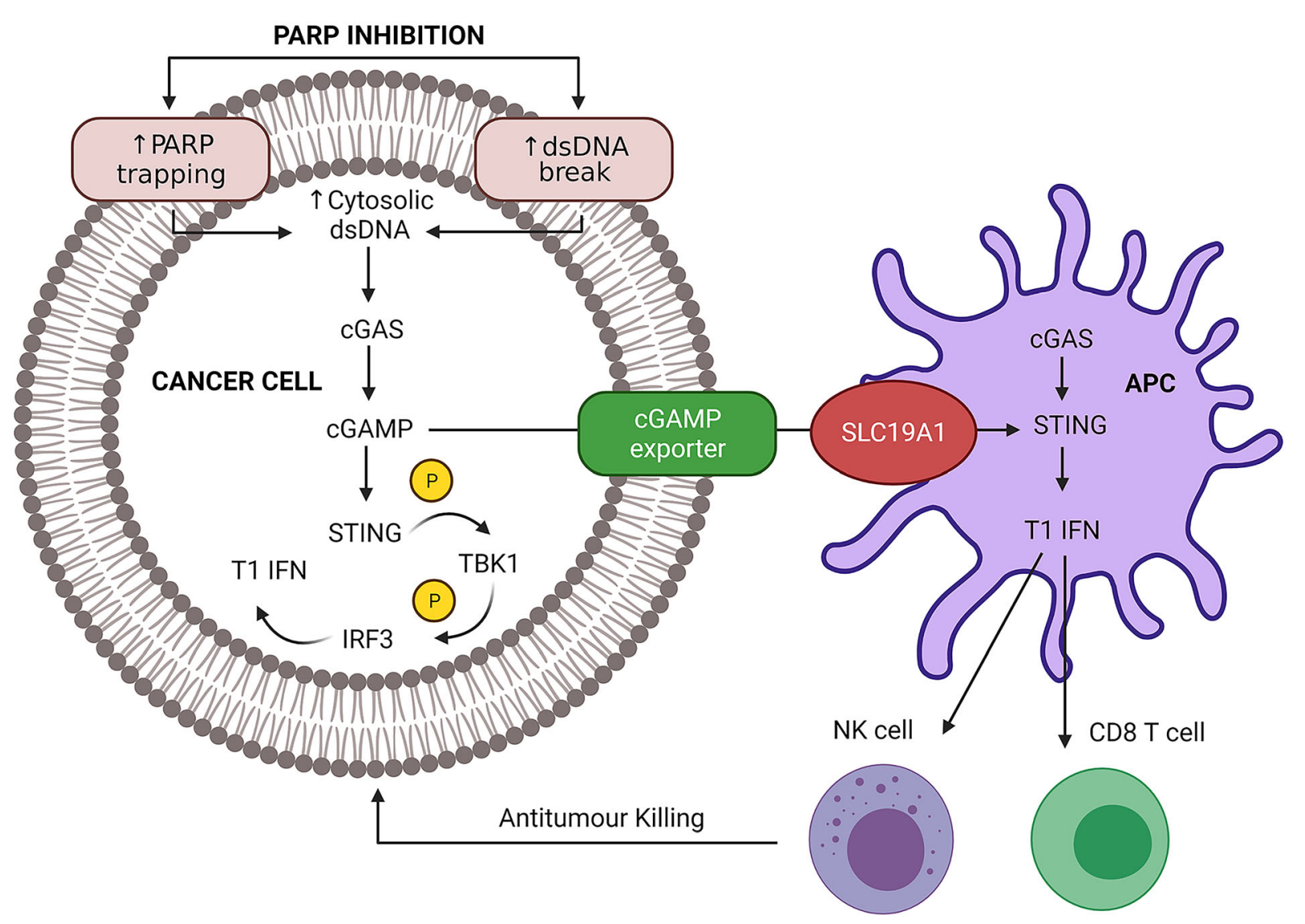

Fig. 6 Schematic depicting activation of cGAS/STING pathway by PARP inhibition, leading to downstream type 1 interferon production resulting in a direct immunosuppressive effect by the cancer cell, as well as increased natural

tumour cells treated with olaparib and investigated the addition of anti-PD-1 antibody. They found that the combination treatment resulted in sustained suppression of BRCA1-deficient tumour cells compared with olaparib alone, concluding that the activation of the PD-1/PDL1 immune-inhibitory pathway induced by PARP inhibition can be overcome by addition of an anti-PD-1 antibody.

A similar mechanism of immunomodulation by PARP inhibition has been demonstrated in other tumour types. In BRCA1-deficient models of triple-negative breast cancer, Pantelidou et al. demonstrated the stimulation by PARPi of CD8+ $T$ cell recruitment mediated via the cGAS/STING pathway in tumour cells, again through activation of host dendritic cells [36]. killer (NK) cell and CD8 cytotoxic T cell activity causing direct anti-tumour killing. IFN interferon, TBK-1 tankbinding kinase 1 , interferon regulatory factor $3, A P C$ antigen-presenting cell

These studies suggest that in the context of HR deficiency, PARP inhibitor-mediated DNA damage can stimulate the STING pathway, converting immunologically cold tumours into hot tumours, providing a good rationale for combining PARPi with immunotherapy.

The TOPACIO trial was a phase $1 / 2$ study evaluating the combination of niraparib and pembrolizumab in patients with metastatic triple-negative breast cancer and platinum-resistant ovarian cancer [37]. Sixty-two patients with ovarian carcinoma were enrolled in phases 1 and 2. The population was diverse with the majority of patients having BRCAwt tumours which were platinum-resistant or platinum-refractory and previous treatment with bevacizumab. Five percent of these 62 patients had a 
CR, $13 \%$ a PR and $47 \%$ stabilisation of disease. In the efficacy evaluable population $(n=60)$, the overall response rate was $18 \%(90 \% \mathrm{CI}$, $11-29 \%)$ and results were similar across the HRD-negative and HRD-positive populations. This response rate is higher than that of patients with BRCAwt and platinum-resistant/refractory disease treated with either single agent PARP inhibitor or single-agent PD-1/PD-L1 inhibitor.

\section{Combinations of Multiple Agents}

In addition to combining PARPi with bevacizumab and PARPi with immunotherapy, there are now studies underway investigating the combination of all three. Preliminary results from the phase 2 OPAL study, which is examining niraparib, bevacizumab and the PD-1 antagonist dostarlimab in recurrent, platinumresistant ovarian cancer, were recently announced at SGO 2021 [38]. Forty-one patients with advanced, recurrent ovarian, fallopian tube or peritoneal cancer were enrolled, 39 of whom were radiologically evaluable. A post hoc analysis for biomarker status revealed that $82.9 \%$ of patients were BRCAwt, $75.6 \%$ HRD, 68.3\% PD-L1-positive and 43.9\% platinum-resistant. The primary end point was objective response rate, which was $17.9 \%$, with seven PRs and no CRs. Twenty-three patients had stable disease, and the overall disease control rate was $76.9 \%$. The median PFS was 7.6 months, and no correlation with biomarker status was seen.

Table 5 presents a list of phase $2 / 3$ clinical trials which are employing the combination of niraparib with other agents in advanced/metastatic epithelial ovarian cancer, and are currently recruiting or have recently completed enrolment, updated as of May 2021 (search terms "Ovarian Cancer" and "Niraparib" on ClinicalTrials.gov).

\section{DISCUSSION}

It is likely that the treatment options for patients with advanced ovarian cancer will continue to expand, with several trials underway exploring different combinations. The best approach will therefore be with individualised treatment regimens to ensure optimum therapy for each patient at the appropriate time. With PARPi licensed in both the front-line and recurrent setting, the decision will need to be made regarding maximising treatment up front or adopting a sequential use of targeted therapies. The concern with 'saving' PARPi for later use in the recurrent setting is that patients will need to demonstrate a second response to platinum-based therapy in order to be eligible, and therefore the opportunity to use PARP inhibition may be lost.

Characterising patients in terms of biomarkers and high-risk features can help with the treatment decision-making process. However, the genomic complexity of epithelial ovarian cancer and the limitations of HRD testing as discussed above have precluded the development of the ultimate biomarker for treatment selection. Establishing a standardised platform for germline testing and tumour sequencing that adequately detects genomic instability to define HRD is essential if it is to be used as a predictive biomarker to guide treatment.

For patients with the BRCA mutation, arguably the best currently licensed up-front treatment is PARP inhibition alone, as these patients are likely to respond very well. Preclinical studies suggest that patients with gBRCA mutations (gBRCA1/2m) have more immunologically inflamed tumours as compared to patients with HRP epithelial ovarian carcinomas [39-41]. Patients with gBRCA1/2m may also have fewer immunosuppressive myeloidderived suppressor cells and more peripheral CD8+ T cells compared to their BRCAwt counterparts. This immune phenotype represents a rich immunological potential that can be harnessed to produce more durable responses. Niraparib, alongside other PARPi, has the potential to activate interferon signalling and enhance tumour neo-antigen presentation to resident tumour infiltration lymphocytes in order to induce immunogenic cell death. Direct STING agonists have been evaluated in the preclinical setting in combination with PARPi. Preliminary in vitro experiments $[26,42]$ of this combination in BRCA-deficient breast cancer 
Table 5 Current phase $2 / 3$ clinical trials of niraparib with other agents in advanced/metastatic epithelial ovarian cancer

\begin{tabular}{|c|c|c|c|c|}
\hline Title & Mechanism & $\begin{array}{l}\text { Current } \\
\text { status }\end{array}$ & Phase & $\begin{array}{l}\text { Target } \\
\text { participants }\end{array}$ \\
\hline $\begin{array}{l}\text { Efficacy and Safety of Niraparib Combined with Oral } \\
\text { Etoposide in Platinum Resistant/Refractory Recurrent } \\
\text { Ovarian Cancer (NCT04217798) }\end{array}$ & $\begin{array}{l}\text { Topoisomerase } \\
\text { inhibitor }\end{array}$ & Recruiting & 2 & $\begin{array}{l}35 \\
\text { participants }\end{array}$ \\
\hline $\begin{array}{l}\text { A Phase } 3 \text { Comparison of Platinum-based Therapy with } \\
\text { TSR-0 } 42 \text { and Niraparib versus SOC Platinum-Based } \\
\text { Therapy as First-Line Treatment of Stage III or IV } \\
\text { Nonmucinous Epithelial Ovarian Cancer (FIRST) } \\
\text { (NCT03602859) }\end{array}$ & Anti-PD-1 inhibitor & $\begin{array}{l}\text { Active, not } \\
\text { recruiting }\end{array}$ & 3 & $\begin{array}{l}1045 \\
\text { participants }\end{array}$ \\
\hline $\begin{array}{l}\text { Anlotinib and Niraparib Dual Therapy Evaluation in } \\
\text { Platinum-resistant Recurrent Ovarian Cancer (ANNIE) } \\
\text { (NCT04376073) }\end{array}$ & $\begin{array}{c}\text { Anti-VEGFR, FGFR, } \\
\text { PDGFR and ckit }\end{array}$ & Recruiting & 2 & $\begin{array}{l}40 \\
\text { participants }\end{array}$ \\
\hline $\begin{array}{l}\text { A Study of Niraparib Combined With Bevacizumab } \\
\text { Maintenance Treatment in Participants With Advanced } \\
\text { Ovarian Cancer Following Response to Front-Line } \\
\text { Platinum-Based Chemotherapy (NCT03326193) }\end{array}$ & Anti-VEGF & $\begin{array}{l}\text { Active, } \\
\text { non- } \\
\text { recruiting }\end{array}$ & 2 & $\begin{array}{l}105 \\
\text { participants }\end{array}$ \\
\hline $\begin{array}{l}\text { Safety and Efficacy of AsiDNATM, a DNA repair } \\
\text { inhibitor, administered IV in addition to niraparib in } \\
\text { patients with relapsed platinum sensitive ovarian cancer } \\
\text { already treated with niraparib (REVOCAN) }\end{array}$ & DNA repair inhibitor & Recruiting & $1 / 2$ & $\begin{array}{l}26 \\
\text { participants }\end{array}$ \\
\hline $\begin{array}{l}\text { Platinum-based Chemotherapy with Atezolizumab and } \\
\text { Niraparib in Patients with Recurrent Ovarian Cancer } \\
\text { (ANITA) }\end{array}$ & $\begin{array}{l}\text { Platinum-based } \\
\text { chemotherapy and } \\
\text { anti-PD-L1 }\end{array}$ & Recruiting & 3 & $\begin{array}{l}414 \\
\text { participants }\end{array}$ \\
\hline $\begin{array}{l}\text { Efficacy and Safety of Niraparib Combined with } \\
\text { Bevacizumab in Platinum Refractory/Resistant Recurrent } \\
\text { Ovarian Cancer (AVANIRA3) }\end{array}$ & Anti-VEGF & Recruiting & 2 & $\begin{array}{l}32 \\
\text { participants }\end{array}$ \\
\hline
\end{tabular}

models demonstrated that PARP inhibition and STING agonism can augment STING pathway activation and pro-inflammatory cytokine production along with improved leucocyte and dendritic cell function compared to monotherapy. Clinical evaluation of direct STING agonism with PARP inhibition is not yet underway, but this is likely to change very soon.

Patients with HRD who are BRCAwt are still likely to experience a good response to PARP inhibition, and monotherapy with niraparib is a reasonable option, especially for those with no residual macroscopic disease post surgery. For those with high-risk features, the addition of bevacizumab to PARPi should be considered, and the combination of olaparib and bevacizumab is licensed in this setting on the basis of the PAOLA-1 trial. There has been no randomised phase 3 trial assessing niraparib with bevacizumab, but the preliminary data from OVARIO are encouraging for this combination as well.

For HRP patients, niraparib monotherapy is currently licensed but is unlikely to be sufficient alone, and combination with bevacizumab is likely to offer the most benefit for these 
patients. In the absence of HRD, platinum sensitivity can be used as a surrogate predictive biomarker for the use of PARP inhibition as well as the presence of high-risk features, as reflected by the patient population in PRIMA. For those with HRP disease who respond less well to platinum-based chemotherapy, perhaps bevacizumab monotherapy should be considered.

To date we have no direct comparison from randomised trials comparing maintenance PARPi with maintenance bevacizumab for platinum-sensitive ovarian cancer. A network metaanalysis of 13 trials (one of which was the NOVA study) involving patients with platinumsensitive recurrent ovarian cancer treated with PARPi or bevacizumab was published last year [43]. These indirect comparisons suggest improved PFS with PARPi compared to bevacizumab, although subgroup analyses indicate that the superiority does not reach statistical significance in the BRCAwt population. Even in this subgroup, however, PARPi maintenance did have the highest likelihood of being ranked as the best treatment according to the surface under the cumulative ranking curve (SUCRA) value. The group therefore concluded that PARPi should be the preferred choice for patients with recurrent platinum-sensitive ovarian cancer. It is important to note that because PARPi are being increasingly used in the first-line setting, we have less knowledge of the role of PARPi for recurrent disease in patients who have already received PARPi up front. Further research is required to assess the efficacy of retreatment with PARPi following relapse.

It is clear that niraparib and other PARPi will continue to play a crucial role in the management of patients with platinum-sensitive epithelial ovarian cancer in both the up-front and recurrent setting, regardless of HR status. Ongoing research is required to improve the outcomes for patients with platinum-resistant disease, as well as to accurately identify a predictive biomarker for PARP inhibition and to maximise the benefit of these agents through combination with other targeted treatments.

\section{ACKNOWLEDGEMENTS}

Figures 1, 4, 5 and 6 are original figures created using Biorender.com.

Funding. No funding or sponsorship was received for this study or publication of this article. However, Rami Mustapha and Kenrick $\mathrm{Ng}$ are currently research fellows funded by Cancer Research UK, under Award Numbers C604/A25135 and C1519/28682 (support of R.M.) and 549580 (support of K.N.).

Authorship. All named authors meet the International Committee of Medical Journal Editors (ICMJE) criteria for authorship for this article, take responsibility for the integrity of the work as a whole, and have given their approval for this version to be published.

Authorship Contributions. Ionut-Gabriel Funingana, Melek Akay, Kenrick Ng and Michael Flynn conceived and designed the review. Melek Akay, Grisma Patel, Rami Mustapha, Ernese Gjafa, Tony $\mathrm{Ng}$ and Kenrick Ng drafted and revised the manuscript. Tony $\mathrm{Ng}$ and Michael Flynn provided supervision of this manuscript. All authors agreed on the final version of the manuscript to be published.

Disclosures. Tony $\mathrm{Ng}$ has received research funding from Astrazeneca and Daichii Sankyo. Kenrick Ng has received honoraria from GSK/ Tesaro, Pfizer and Boehringer Ingelheim and travel expenses from GSK/Tesaro. Michael Flynn has received honoraria from GSK. Ionut Gabriel Funingana has received travel expenses from GSK/TESARO. The other authors declare no conflicts of interests.

Compliance with Ethics Guidelines. This article is based on previously conducted studies and does not contain any studies with human participants or animals performed by any of the authors.

Data Availability. Data sharing is not applicable to this article as no data sets were generated or analysed during the current study. 
Open Access. This article is licensed under a Creative Commons Attribution-NonCommercial 4.0 International License, which permits any non-commercial use, sharing, adaptation, distribution and reproduction in any medium or format, as long as you give appropriate credit to the original author(s) and the source, provide a link to the Creative Commons licence, and indicate if changes were made. The images or other third party material in this article are included in the article's Creative Commons licence, unless indicated otherwise in a credit line to the material. If material is not included in the article's Creative Commons licence and your intended use is not permitted by statutory regulation or exceeds the permitted use, you will need to obtain permission directly from the copyright holder. To view a copy of this licence, visit http://creativecommons.org/licenses/by$\mathrm{nc} / 4.0 /$.

\section{REFERENCES}

1. Hoeijmakers JH. Genome maintenance mechanisms for preventing cancer. Nature. 2001;411(6835):366-74.

2. Lord CJ, Ashworth A. PARP inhibitors: Synthetic lethality in the clinic. Science. 2017;355(6330): 1152-8.

3. Huber A, Bai P, de Murcia JM, de Murcia G. PARP-1, PARP-2 and ATM in the DNA damage response: functional synergy in mouse development. DNA Repair (Amst). 2004;3(8-9):1103-8.

4. Hopkins TA, Ainsworth WB, Ellis PA, et al. PARP1 Trapping by PARP Inhibitors Drives Cytotoxicity in Both Cancer Cells and Healthy Bone Marrow. Mol Cancer Res. 2019;17(2):409-19.

5. Nijman SM, Friend SH. Potential of the synthetic lethality principle. Science. 2013;342(6160): 809-11.

6. Sandhu SK, Schelman WR, Wilding G, et al. The poly(ADP-ribose) polymerase inhibitor niraparib (MK4827) in BRCA mutation carriers and patients with sporadic cancer: a phase 1 dose-escalation trial. Lancet Oncol. 2013;14(9):882-92.

7. Jones P, Altamura S, Boueres J, et al. Discovery of 2-\{4-[(3S)-piperidin-3-yl]phenyl\}-2H-indazole-7-carboxamide (MK-4827): a novel oral poly(ADP- ribose)polymerase (PARP) inhibitor efficacious in BRCA-1 and -2 mutant tumors. J Med Chem. 2009;52(22):7170-85.

8. Jones P, Wilcoxen $\mathrm{K}$, Rowley $\mathrm{M}$, Toniatti C. Niraparib: A Poly(ADP-ribose) Polymerase (PARP) Inhibitor for the Treatment of Tumors with Defective Homologous Recombination. J Med Chem. 2015;58(8):3302-14.

9. Mikule KWK. Abstract B168: The PARP inhibitor niraparib crosses the blood brain barrier in rodents and is efficacious in a BRCA2 mutant intracranial tumor model. Mol Cancer Ther. 2015;14(12):168.

10. Mirza MR, Monk BJ, Herrstedt J, et al. Niraparib Maintenance Therapy in Platinum-Sensitive, Recurrent Ovarian Cancer. N Engl J Med. 2016;375(22):2154-64.

11. Mirza MR, Benigno B, Dørum A, et al. Long-term safety in patients with recurrent ovarian cancer treated with niraparib versus placebo: Results from the phase III ENGOT-OV16/NOVA trial. Gynecol Oncol. 2020;159(2):442-8.

12. LaFargue CJ, Dal Molin GZ, Sood AK, Coleman RL. Exploring and comparing adverse events between PARP inhibitors. Lancet Oncol. 2019;20(1):e15-28.

13. Berek JS, Matulonis UA, Peen U, et al. Safety and dose modification for patients receiving niraparib. Ann Oncol. 2018;29(8):1784-92.

14. Oza AM, Matulonis UA, Malander S, et al. Quality of life in patients with recurrent ovarian cancer treated with niraparib versus placebo (ENGOT-OV16/ NOVA): results from a double-blind, phase 3 , randomised controlled trial. Lancet Oncol. 2018;19(8): 1117-25.

15. Gonzalez-Martin A, Pothuri B, Vergote I, et al. Niraparib in Patients with Newly Diagnosed Advanced Ovarian Cancer. N Engl J Med. 2019;381(25):2391-402.

16. Moore KN, Secord AA, Geller MA, et al. Niraparib monotherapy for late-line treatment of ovarian cancer (QUADRA): a multicentre, open-label, single-arm, phase 2 trial. Lancet Oncol. 2019;20(5): 636-48.

17. Telli ML, Timms KM, Reid J, et al. Homologous Recombination Deficiency (HRD) Score Predicts Response to Platinum-Containing Neoadjuvant Chemotherapy in Patients with Triple-Negative Breast Cancer. Clin Cancer Res. 2016;22(15): 3764-73.

18. Miller RE, Leary A, Scott CL, et al. ESMO recommendations on predictive biomarker testing for homologous recombination deficiency and PARP 
inhibitor benefit in ovarian cancer. Ann Oncol. 2020;31(12):1606-22.

19. Burger RA, Brady MF, Bookman MA, et al. Incorporation of bevacizumab in the primary treatment of ovarian cancer. N Engl J Med. 2011;365(26): 2473-83.

20. Perren TJ, Swart AM, Pfisterer J, et al. A phase 3 trial of bevacizumab in ovarian cancer. N Engl J Med. 2011;365(26):2484-96.

21. Elaimy $\mathrm{AL}$, Amante JJ, Zhu LJ, et al. The VEGF receptor neuropilin 2 promotes homologous recombination by stimulating YAP/TAZ-mediated Rad51 expression. Proc Natl Acad Sci U S A. 2019;116(28):14174-80.

22. Ray-Coquard I, Pautier P, Pignata S, et al. Olaparib plus Bevacizumab as First-Line Maintenance in Ovarian Cancer. N Engl J Med. 2019;381(25): 2416-28.

23. Mirza MR, Avall Lundqvist E, Birrer MJ, et al. Niraparib plus bevacizumab versus niraparib alone for platinum-sensitive recurrent ovarian cancer (NSGO-AVANOVA2/ENGOT-ov24): a randomised, phase 2, superiority trial. Lancet Oncol. 2019;20(10):1409-19.

24. Hardesty MM KT, Wright GS et al. Phase 2 OVARIO study of niraparib plus bevacizumab therapy in advanced ovarian cancer following frontline platinum-base chemotherapy with bevacizumab. Society of Gynecologic Oncology Virtual Annual Meeting on Women's Cancer. 2021.

25. Borella F, Ghisoni E, Giannone G, et al. Immune Checkpoint Inhibitors in Epithelial Ovarian Cancer: An Overview on Efficacy and Future Perspectives. Diagnostics (Basel). 2020;10(3):146.

26. Constantia Pantelidou HJ, Aditi Kothari, Renyan Liu, Jennifer L. Guerriero, Geoffrey I. Shapiro. STING agonism enhances anti-tumor immune responses and therapeutic efficacy of PARP inhibition in BRCA-associated breast cancer. bioRxiv. 2021.

27. Du H, Xu T, Cui M. cGAS-STING signaling in cancer immunity and immunotherapy. Biomed Pharmacother. 2021;133:110972.

28. Kim C, Wang XD, Yu Y. PARP1 inhibitors trigger innate immunity via PARP1 trapping-induced DNA damage response. Elife. 2020;9:e60637.

29. Zheng J, Mo J, Zhu T, et al. Comprehensive elaboration of the cGAS-STING signaling axis in cancer development and immunotherapy. Mol Cancer. 2020;19(1):133.
30. Kwon J, Bakhoum SF. The Cytosolic DNA-Sensing cGAS-STING Pathway in Cancer. Cancer Discov. 2020;10(1):26-39.

31. Kitai Y, Kawasaki T, Sueyoshi T, et al. DNA-containing exosomes derived from cancer cells treated with topotecan activate a STING-dependent pathway and reinforce antitumor immunity. J Immunol. 2017;198(4):1649-59.

32. Ablasser A, Schmid-Burgk JL, Hemmerling I, et al. Cell intrinsic immunity spreads to bystander cells via the intercellular transfer of cGAMP. Nature. 2013;503(7477):530-4.

33. Jiao S, Xia W, Yamaguchi $\mathrm{H}$, et al. PARP Inhibitor Upregulates PD-L1 Expression and Enhances Cancer-Associated Immunosuppression. Clin Cancer Res. 2017;23(14):3711-20.

34. Wang Z, Sun K, Xiao Y, et al. Niraparib activates interferon signaling and potentiates anti-PD-1 antibody efficacy in tumor models. Sci Rep. 2019;9(1):1853.

35. Ding L, Kim HJ, Wang Q et al. PARP inhibition elicits STING-dependent antitumor immunity in Brca1-deficient ovarian cancer. Cell Rep. 2018;25(11):2972-2980 e2975.

36. Pantelidou C, Sonzogni O, De Oliveria TM, et al. PARP Inhibitor Efficacy Depends on CD8(+) T-cell Recruitment via Intratumoral STING Pathway Activation in BRCA-Deficient Models of TripleNegative Breast Cancer. Cancer Discov. 2019;9(6): 722-37.

37. Konstantinopoulos PA, Waggoner S, Vidal GA, et al. Single-Arm Phases 1 and 2 Trial of Niraparib in Combination With Pembrolizumab in Patients With Recurrent Platinum-Resistant Ovarian Carcinoma. JAMA Oncol. 2019;5(8):1141-9.

38. Liu JF GS, Wahner Hendrickson AE, et al. An openlabel phase II study of dostarlimab (TSR-042), bevacizumab (bev), and niraparib combination in patients (pts) with platinum-resistant ovarian cancer (PROC): Cohort A of the OPAL trial. Society of Gynecologic Oncology 2021 Virtual Annual Meeting on Women's Cancer. March 2021.

39. Koti M, Siu A, Clément I, et al. A distinct pre-existing inflammatory tumour microenvironment is associated with chemotherapy resistance in highgrade serous epithelial ovarian cancer. Br J Cancer. 2015;112(7):1215-22.

40. $\mathrm{Xu} \mathrm{H}, \mathrm{Xian} \mathrm{J}$, Vire $\mathrm{E}$, et al. Up-regulation of the interferon-related genes in BRCA2 knockout epithelial cells. J Pathol. 2014;234(3):386-97. 
41. Strickland KC, Howitt BE, Shukla SA, et al. Association and prognostic significance of BRCA1/2-mutation status with neoantigen load, number of tumor-infiltrating lymphocytes and expression of PD-1/PD-L1 in high grade serous ovarian cancer. Oncotarget. 2016;7(12):13587-98.

42. Jeyasekharan AD. PARP inhibitors need an extra STING for therapeutic efficacy. Sci Transl Med. 2019;11(494).

43. Bartoletti M, Pelizzari G, Gerratana L, et al. Bevacizumab or PARP-inhibitors maintenance therapy for platinum-sensitive recurrent ovarian cancer: a network meta-analysis. Int J Mol Sci. 2020;21(11). 